\title{
An Efficient Protocol for the Production of Pure Lines in Flowering Chinese Cabbage
}

\author{
Yan Yao, Yao Kong, and Ping Zhang ${ }^{1}$
}

School of Life Science, Key Laboratory for Functional Study on Plant StressResistant Genes, Guangzhou University, Guangzhou University City Outer Ring Road 230, Guangzhou 510006, China

\section{Hua Zhang, Hong-di Huang, and Guang-guang Li \\ Guangzhou Institute of Agricultural Sciences, East Xingang Road 151, Guangzhou 510000, China}

Additional index words. Brassica rapa ssp. chinensis var. utilis Tsen et Lee, embryo culture, short generation cycle, recombinant inbred lines, single seed descent

\begin{abstract}
The rapid expansion of Asian populations in the United States presents significant requirements for Asian vegetables. Flowering chinese cabbage (Brassica rapa L. ssp. chinensis var. utilis Tsen et Lee) is one of the most popular vegetables in China. The main factors restricting the progress in its breeding and genetic studies is the time required in generating desired pure line populations. Doubled haploid (DH) populations of flowering chinese cabbage have not been established because of technical difficulties. An appropriate combined protocol for a fast generation cycling system could advance up to seven generations, allowing the production of pure line seeds within 336-420 days among four cultivars and one hybrid of flowering chinese cabbage. The previous six generation cycles were accelerated using the embryo culture plus soil method which bypassed seed maturation through in vitro culture of immature embryos and promoted plant reproduction under stressed conditions, then the seventh generation cycle was accomplished until mature seeds were harvested using the soil method. During the culture of immature embryos, 12-day-old embryos could germinate and develop successfully on a Murashige and Skoog medium (MS) medium (Murashige and Skoog, 1962) containing $10 \%$ young coconut juice. This combined protocol bypasses the current obstacles in constructing DH populations of flowering chinese cabbage and is a possible alternative for producing pure lines. Its wider adoption could facilitate the breeding and biological studies of other Brassicaceae vegetables.
\end{abstract}

Chinese cabbage (Brassica rapa $\mathrm{L}$.) is an important cruciferous vegetable, which has been grown in Asia since the fifth century. The rapid expansion of Asian populations in the United States presents significant opportunities for the U.S. production sector to supply new ethnic vegetables to engage in these emerging markets (Mou and Wang, 2012; Sciarappa et al., 2016). The leading Chinese vegetable in the United States has been bok choy (Sciarappa et al., 2016). Bok

Received for publication 2 Feb. 2017. Accepted for publication 15 May 2017.

Financial support from Guangdong Province Science and Technology Project (2015A020209191), National Natural Science Foundation of China (41373118), and Guangzhou Education Bureau Innovation Team Project (No. 13C02) are appreciated. We are grateful to $\mathrm{H}$. Liu for reviewing this article and providing valuable comments.

Author contributions: Y.Y., Y.K., H.Z., D.H., and G.L. conducted experimental design, data analysis, and manuscript writing. P.Z. was involved in strategic experimental input. Y.Y. and Y.K. conducted the experiments. Y.Y. drafted the manuscript and P.Z. revised and finalized the manuscript.

${ }^{1}$ Corresponding author. E-mail: zip514@163. com.
Choy (B. rapa L., Chinensis group) is one group of chinese cabbage (B. rapa L.) (Stephens, 2015). Flowering chinese cabbage (Brassica rapa L. ssp. chinensis var. utilis Tsen et Lee) is a green cultivar of the flowering type of bok choy and one of most important special vegetables in China. To satisfy the requirements for the breeding and genetic studies, a fast pure-line production procedure might prove important.

Pure-line segregating populations are essential for plant breeding and genetic studies. Doubled haploid lines and recombinant inbred lines (RIL) are the most commonly used "pure lines." The DH approach is the fastest route to obtain homozygous lines so far (Chase, 1952; Ferrie and Mollers, 2010). However, B. rapa is deemed a recalcitrant species in cell and tissue culture (Ajisaka et al., 1999; Sato et al., 1989; Shumilina et al., 2015; Yang et al., 2013). Especially with flowering chinese cabbage, DHs have not been established successfully because of technical difficulties in anther and microspore culture of the species (Li et al., 2011; Zhu et al., 2008).

For production of RILs, individual plants of an $\mathrm{F}_{2}$ population must be self-pollinated for at least 5-6 generations via single-seed descent (SSD) to achieve the desired level of homozygosity. This process takes several years and is therefore a critical factor limiting the efficiency of breeding and genetic studies.

The single-seed descent approach is one method for advancing generations. A fast generation strategy to force flower differentiation and to culture young embryos in vitro dramatically shortened the life cycles in protein legumes, wheat, barley, oat, and triticale (Liu et al., 2016; Ochatt et al., 2002; Zheng et al., 2013). Environmental factors, such as spectral composition of radiation, photoperiod length, growth temperature, and stresses, play a key role in the regulation of the transition to flowering in plants (Ribalta et al., 2014). In addition to the management of environmental factors to accelerate flowering, the technique of young embryo culture in vitro has also been used to shorten the lifecycles by circumventing the requirements of seed maturation and postharvest dormancy (Mobini and Warkentin, 2016; Zheng et al., 2013). The suitable embryo ages and medium content have important effects on the success of embryo culture. Meanwhile, the previous reports of embryo culture in different species were variation. The genetically distant hybrid embryos $10 \mathrm{~d}$ post pollination in Brassica species needed three different media to be cultured until seedling stage (Wang et al., 2010). Embryos 12-14 d post pollination of wheat, barley, oat, and triticale could germinate on an MS medium containing young coconut juice (Liu et al., 2016; Zheng et al., 2013). Mobini and Warkentin (2016) reported that young embryos 13$17 \mathrm{~d}$ post pollination for pea could germinate on a Holland Secret medium. They established an efficient method for pea and examined two pea RIL populations for seven generations using the method. However, embryo culture of flowering chinese cabbage was seldom reported (Li et al., 2011).

The main objective of this study was to establish an efficient protocol for the production of pure lines of flowering chinese cabbage (Brassica rapa L. ssp. chinensis var. utilis Tsen et Lee) to bypass the obstacles in constructing DHs. We compared two methods, soil method and embryo culture plus soil method, to affect one generation cycle and tested seven generation cycles in four cultivars and one hybrid of flowering chinese cabbage.

\section{Materials and Methods}

Plant materials. The standard early ('Sijiu 19' and 'Lvyou 501'), medium ('Lvbao 70'), and late flowering cultivars ('Chi 802'), and one hybrid (Sijiu $19 \times 3$ T-6) were used in this study (The plants of flowering chinese cabbage growing in a natural field are showed in Fig. 1A). Two methods (detailed below) were investigated for the plant fast generation. For each cultivar and hybrid, twelve plants were grown as replicates in each growing condition.

Soil method. Plants were grown in soil for the whole generation from seed germination 
to seed production for the next generation. Seeds were directly sown in $4 \times 8$ (32-well) Kwikpot trays. The volume of each of the wells was $\approx 100 \mathrm{~cm}^{3}$ for the 32 well trays containing steam-sterilized potting mix $(50 \%$ sand and $50 \%$ peat $\mathrm{v} / \mathrm{v}$ ). Trays were arranged in a controlled environment room set at $25 / 22$ $( \pm 1){ }^{\circ} \mathrm{C}$ day/night temperature and $65 \% / 85 \%$ day/night relative humidity, and a $24-\mathrm{h}$ photoperiod with light intensity of $500 \mu \mathrm{mol} \cdot \mathrm{m}^{-2} \cdot \mathrm{s}^{-1}$ photon flux density at the canopy level. White fluorescent tubes were used as light resources. To promote plant development, water stress was applied with plants watered only when clear water-stress symptom appeared. During flowering, artificial self-pollination was needed to improve seed set. The first flower was manually self-pollinated, and the remaining flowers were removed to keep a single pod per plant (Fig. 1B). Seeds were harvested $30 \mathrm{~d}$ after flowering or pollination and then dried at $37{ }^{\circ} \mathrm{C}$ for $5 \mathrm{~d}$ before sowing for the next generation. The duration of one generation was measured from seed sowing of the previous generation to seed sowing of the next generation.

Embryo culture plus soil method. Plants of the first generation were grown in soil. Light, temperature, and irrigation were as per the soil method. The date to first flower was recorded for each plant. The first flower was manually self-pollinated, and the remaining flowers were removed to keep a single pod per plant. The embryos were dissected from the developing seeds $10,12,16,20,24$, and $28 \mathrm{~d}$ after pollination and then cultured in vitro. Initially, only early flowering cultivar Sijiu 19 and late flowering cultivar Chi 802 were tested to develop the embryo culture protocol and then the same developed optimum protocol was further tested on the other genotypes. The method was conducted as explained below.

Embryo culture. A series of preliminary experiments were conducted to determine the optimum conditions for embryo culture to obtain new generation seedlings within the shortest timeframe. Factors investigated include 1) age of embryos measured by days post anthesis (dpa), and 2) time taken to produce embryo-generated seedlings for transferring to potting mix, measured by days post embryo culture (dpec).

Young pods of flowering chinese cabbage were surface sterilized with $1 \%$ sodium hypochlorite solution for $10 \mathrm{~min}$; the sterilized pods were then rinsed three times with sterilized distilled water. Young seeds were removed from the pods, and the embryos were carefully dissected under aseptic conditions and placed on an MS medium (Murashige and Skoog, 1962) containing $10 \%$ young coconut juice (Thai World Import \& Export Co., Ltd, Bangkok, Thailand) and $1 \%$ sucrose. Preparation of reagents and media were the same as described in Zheng et al. (2013). Petri dishes containing newly cultured embryos were kept at $25{ }^{\circ} \mathrm{C}$ in a dark incubator. When the cultured embryos started to germinate, the plates were transferred into a constant temperature room with 16-h light period and $25{ }^{\circ} \mathrm{C} / 22{ }^{\circ} \mathrm{C}$ day/night temperatures. When the roots of seedlings were about $1.5-2.0 \mathrm{~cm}$ long, they were transferred into Kwikpot trays to start the next generation in soil. The duration of one generation was measured from embryo culture of the previous generation to embryo culture of the next generation.

Production of pure lines. The combinations of treatments from the preliminary experiments were applied on four cultivars and one hybrid in the development of seven generations for evaluation of the final combined protocol. For each cultivar and hybrid, 12 plants were grown as replicates. The first generation was conducted using the soil method from the seeds sowing to the embryos culture and then the second generation was started from the first generation embryo culture to the second generation embryo culture using the embryo culture plus soil method. The other generation cycles were the same as the second generation cycle except that the period of the final seventh generation was from embryo culture to harvesting of seeds using the soil method.

Statistical analysis. The experimental data were subjected to statistical analysis and SD was computed using the software SPSS (Version 16.0) developed by IBM, USA.

\section{Results}

One generation cycle using the soil method. The number of days from sowing to flowering in the soil method with single pod ranged from 30.4 to $42.6 \mathrm{~d}$ for the four cultivars and one hybrid (Table 1). 'Sijiu 19' flowered first whereas 'Chi 802' was the last to flower.

From flowering to seed harvest, an average of 35 dpa was needed with no distinct difference among cultivars. The one generation cycle for the soil method with the "single pod" ranged from 65 to $77 \mathrm{~d}$. The earlyflowering genotypes, Sijiu 19, hybrid (Sijiu $19 \times 3$ T-6) and Lvyou 501, took 65, 66, and $67 \mathrm{~d}$, respectively. The medium-flowering cultivar Lvbao 70 took $72 \mathrm{~d}$, and the late cultivar Chi 802 took $77 \mathrm{~d}$. Furthermore, the average number of seeds per pod produced with this method was $\approx 3-5$ seeds (Table 1 ).

Effect of embryo age on embryo germination using the embryo culture plus soil method. In this treatment of the early-flowering cultivar
Sijiu 19, the older the embryos were, the less time that was needed for the embryos to germinate. Embryos less than 10-d old (Fig. 2A) were too young to germinate; and embryos that were 12-d old (Fig. 2B) spent $7.2 \mathrm{~d}$ from transferring embryos on media to germinating seedling. Fewer days were required for the embryos over 12-d old, including those 20- and 28-d old (Fig. 2C-E; Table 2). Twenty-eight-day old embryos only spent $4.5 \mathrm{~d}$ to get suitable seedling for the next generation (Table 2). To realize the shortest generation time considering both embryo ages and embryo-germinated times, 12-d-old embryos spent the least time of about $46 \mathrm{~d}$ for one generation from seed sowing of the first generation to the seedling stage of next generation (Fig. $2 \mathrm{~F}$ and $\mathrm{G}$; Table 2). Although the late-flowering cultivar Chi 802 spent more days from sowing to flowering, the earliest optimum embryo culture age (12 dpa), and the shortest embryogerminated time ( $6.8 \mathrm{~d})$ were almost the same as for 'Sijiu 19' (Table 2).

One generation cycle of different cultivars using the embryo culture plus soil method. To verify the feasibility of the embryo culture plus soil method, three cultivars (Lvyou 501, Lvbao 70, and Chi 802) and one hybrid (Sijiu $19 \times 3$ T-6) were also tested using this method. The results showed that the embryo culture age of $12-\mathrm{d}$ old and the embryo-germinated time of $7 \mathrm{~d}$ could apply to these tested materials. Using the embryo culture plus soil method, the one generation cycle of 'Sijiu 19', 'Lvyou 501', and hybrid (Sijiu $19 \times 3$ T-6) took 46, 48, and $47 \mathrm{~d}$, respectively. The medium-flowering cultivar Lvbao 70 took $53 \mathrm{~d}$, and the late cultivar Chi 802 took $58 \mathrm{~d}$. Compared with the soil

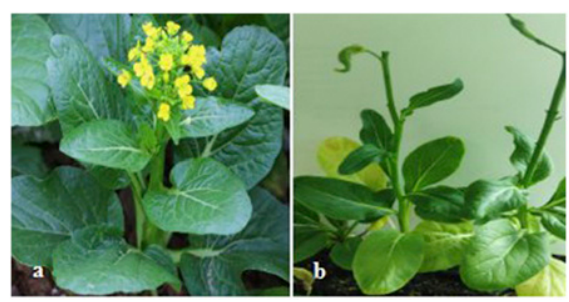

Fig. 1. Plants of flowering chinese cabbage. (A) Plants growing in natural condition and (B) plants growing in Kwikpot tray with a single pod.

Table 1. One generation cycle using the soil method.

\begin{tabular}{|c|c|c|c|c|c|}
\hline Variety & Flowering type & $\begin{array}{l}\text { Days from } \\
\text { sowing to } \\
\text { flowering }\end{array}$ & $\begin{array}{l}\text { Days from flowering } \\
\text { to seed harvested } \\
\text { plus drying time }\end{array}$ & $\begin{array}{l}\text { One cycle } \\
\text { time }(d)^{x}\end{array}$ & Seeds per pod ${ }^{\mathrm{z}}$ \\
\hline Sijiu 19 & Early & $30.4 \pm 1.9$ & 35 & 65 & $4.3 \pm 2.0$ \\
\hline Lvyou 501 & Early & $32.2 \pm 2.3$ & 35 & 67 & $5.4 \pm 1.9$ \\
\hline Lvbao 70 & Medium & $37.3 \pm 2.8$ & 35 & 72 & $5.0 \pm 2.1$ \\
\hline Chi 802 & Late & $42.6 \pm 3.5$ & 35 & 77 & $3.6 \pm 2.3$ \\
\hline Sijiu $19 \times 3$ T -6 & & $31.4 \pm 4.2$ & 35 & 66 & $4.0 \pm 1.7$ \\
\hline
\end{tabular}

${ }^{\mathrm{z}}$ Mean $\pm \mathrm{SD}$

yPlants using the soil method usually spent $30 \mathrm{~d}$ from flowering to seed harvested, then extra $5 \mathrm{~d}$ needed to dry the seeds.

${ }^{\mathrm{x}}$ One cycle time: the whole generation from seed sowing to the next generation seed sowing. 
method, the embryo culture plus soil method effectively shortened $19 \mathrm{~d}$ of one generation cycle.

An efficient combined protocol for the production of pure lines. To obtain the seeds of pure lines, the four cultivars and one hybrid (Sijiu $19 \times 3$ T-6) were grown until the seventh generation using a combined

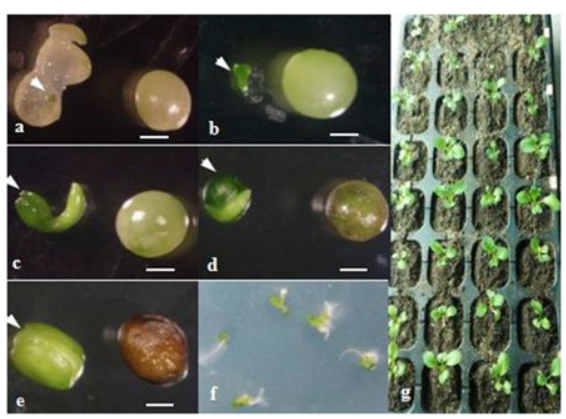

Fig. 2. Developmental stages of young seeds and embryos of flowering chinese cabbage (bars $=1$ $\mathrm{mm}$ ): young embryos (on the left; arrows indicated the young embryos) dissected from young seeds or mature seeds (on the right) at (A) 10-d old; (B) 12-d old; (C) 16-d old; (D) 20-d old and (E) 28-d old; (F) germinated embryos on the medium; and $(\mathbf{G})$ the next generation plantlets derived from embryo culture.

Table 2. Effect of embryo age of 'Sijiu 19' and 'Chi 802' on embryo germination using the embryo culture plus soil method.

\begin{tabular}{|c|c|c|c|c|c|c|}
\hline \multirow[b]{2}{*}{$\begin{array}{l}\text { Embryo age } \\
(\text { dpa })^{z}\end{array}$} & \multicolumn{3}{|c|}{ Sijiu 19} & \multicolumn{3}{|c|}{ Chi 802} \\
\hline & $\begin{array}{c}\text { Days post } \\
\text { embryo culture } \\
(\text { dpec })^{\mathrm{y}}\end{array}$ & $\begin{array}{c}\text { Embryo } \\
\text { germination } \\
\text { rate }(\%)\end{array}$ & $\begin{array}{c}\text { One cycle } \\
\text { time }(d)^{x}\end{array}$ & $\begin{array}{c}\text { Days post } \\
\text { embryo culture } \\
(\text { dpec })^{\mathrm{y}}\end{array}$ & $\begin{array}{c}\text { Embryo } \\
\text { germination } \\
\text { rate }(\%)\end{array}$ & $\begin{array}{l}\text { One cycle } \\
\text { time }(d)^{x}\end{array}$ \\
\hline 10 & $13.5 \pm 2.6$ & 33.3 & 51 & $11.8 \pm 0.7$ & 16.7 & 61 \\
\hline 12 & $7.2 \pm 2.2$ & 100 & 46 & $6.8 \pm 2.3$ & 83.3 & 58 \\
\hline 16 & $8.1 \pm 2.8$ & 100 & 51 & $7.4 \pm 1.0$ & 100 & 62 \\
\hline 20 & $6.8 \pm 2.4$ & 100 & 54 & $5.9 \pm 2.8$ & 100 & 64 \\
\hline 24 & $5.2 \pm 1.4$ & 100 & 56 & $4.8 \pm 0.9$ & 100 & 67 \\
\hline 28 & $4.5 \pm 0.5$ & 100 & 59 & $4.1 \pm 2.4$ & 83.3 & 71 \\
\hline
\end{tabular}

${ }^{\mathrm{z}}$ Embryo age (dpa): days post anthesis.

${ }^{y}$ Days post embryo culture (dpec): days required for embryo culture before transferring into potting mix $($ mean $\pm \mathrm{SD})$.

${ }^{\mathrm{x}}$ One cycle time: measured from embryo culture of this generation to embryo culture of the next generation.

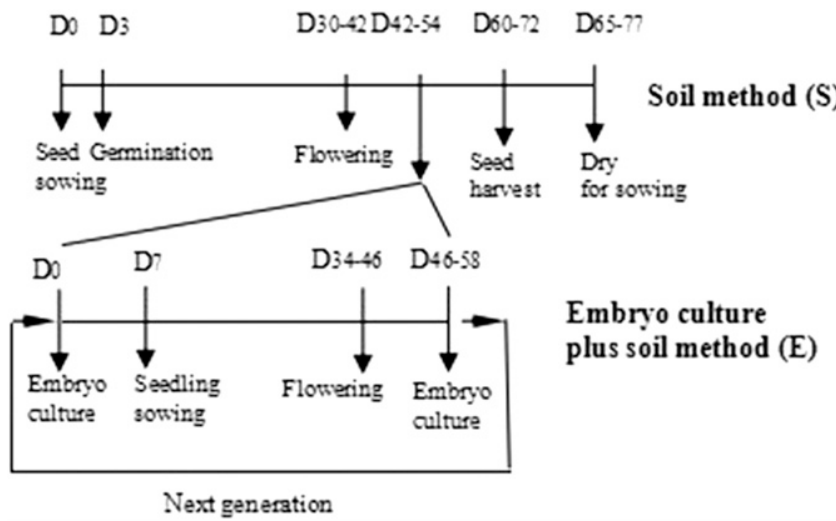

Fig. 3. Timelines for days (D) of one generation cycle for flowering chinese cabbage using the soil method (S) and the embryo culture plus soil method (E). bypassed seed maturation through in vitro culture of immature embryos, and the last generation cycle was accomplished using the soil method. All of the tested materials spent 336-420 d to complete seven generation cycles. This efficient combined protocol makes it possible to produce pure lines seeds of flowering chinese cabbage within $1-1.2$ years.

One generation cycle from seed to seed of flowering chinese cabbage planting in the field takes $90-150 \mathrm{~d}$ in south China. Using the embryo culture plus soil method, only 46-58 d are needed in one generation cycle. It is highly efficient to shorten the breeding time. Although the embryo culture process needs skilled personnel to operate, it is relatively easy to learn and is not very labor intensive as the culture only involves embryo germination step. To develop one population with 200 individuals for seven generation cycles, 200 embryos will be dissected and cultured on media for each generation. It is easy to fulfill in one day for a skilled person who usually operate 200-250 embryos per day from pod surface sterilization to placing embryos on media. Another $2 \mathrm{~d}$ are necessary to prepare media and screen immature seeds. Totally, $3 \mathrm{~d}$ are enough for one person to conduct one generation embryo culture of one population. Therefore, only one skilled person can operate the embryos culture for nearly 10 15 RILs during one generation cycle in 60 d. The method of embryo culture does not need more personnel than the soil method from seed by seed.

In the embryo culture plus soil method, medium content has important effects on the success of embryo culture. Compared with the report of three different media needed for the distantly related hybrid embryos of Brassica species from embryo to seedling (Wang et al., 2010), the MS medium containing $10 \%$ young coconut juice of this study could induce embryo germination of flowering chinese cabbage directly. The component of this medium is simple and also suitable for the embryo culture of other species, such as wheat, barley, oat, and triticale (Liu et al., 2016; Zheng et al., 2013). This embryo culture medium probably has extensive applicability for embryo culture using different species. Besides the embryo culture medium, embryo age is important as well. In this study, 12-d-old embryos were the youngest ones used for culture, and use of these resulted in the least time for one generation cycle. Meanwhile, many factors could influence embryo and plant development, including environmental factors such as light intensity and duration, temperature and watering regimes, and the number of pods retained on the plant. Higher temperature and higher light intensity would increase stress level and therefore shorten the generation cycle but also with greater risk of no seed production. If plants are grown in less favorable environments than that used in this study, embryos more than 12-d old might be needed. Otherwise, younger embryos were difficult to germinate. 


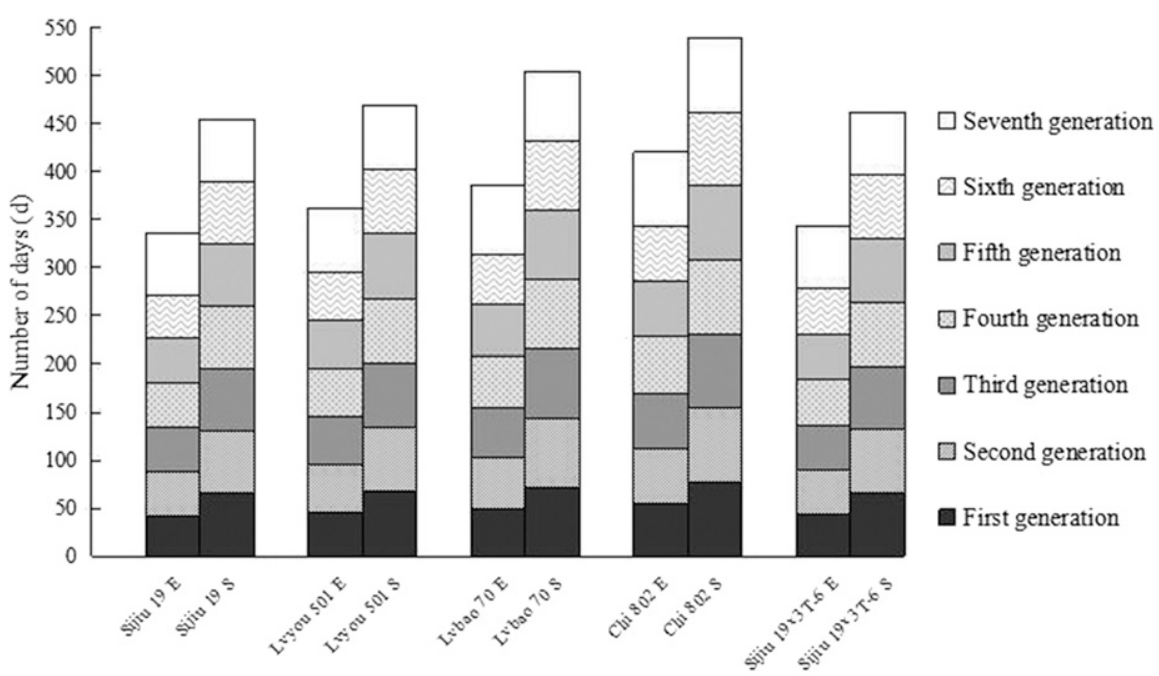

Fig. 4. Different lengths of seven generation cycles using the embryo culture plus soil method (E) and the soil method $(\mathrm{S})$ in four varieties and one hybrid of flowering chinese cabbage.

The embryo culture plus soil method will require less time to advance the generation cycle than the soil method, but both of them need a controlled environment chamber to create stressed conditions for accelerating the development of plants. It is a fact that running the controlled environment chamber will increase the cost of operations. However, the chamber can be used efficiently. The trays holding planted seedlings were placed on a shelf with four layers covering about $1 \mathrm{~m}^{2}$ area, which can grow 450-500 plants, enough for 2-3 populations if each population contains 150-200 individuals. Usually, 25-30 populations can be cultured in a $10-15 \mathrm{~m}^{2}$ controlled environment chamber at the same time.

Flowering chinese cabbage is considered a crosspollinated crop with $80 \%$ to $100 \%$ crosspollination rate in the open field (Zhang et al., 2002). To improve self-pollination rate of SSD in this experiment, manual selfpollination is needed to produce viable seeds in pods. In this study, the first strong flower on each plant was retained and manual selfpollination was conducted, a single pod with 3-5 seeds or embryos could be harvested from each plant. By the SSD approach, we selected the biggest seed in the pod of each plant, which were reliable to continue the new cycle. If a larger number of progeny for each generation is required, a slightly larger volume of tray cell/pot or 2-3 being kept per plant would be necessary to produce more seeds for each line (Liu et al., 2016).

The embryo culture plus soil method outlined herein to accelerate the prior six generation cycles and used with the soil method to harvest seeds of the seventh generation cycle, provides an efficient protocol that can advance the SSD procedure and produce pure lines seeds of flowering chinese cabbage within $1-1.2$ years. Meanwhile, it is important to note that the soil method also can be used in situations where skilled personnel and embryo culture facilities are not available. The generation cycle in this case is from seed to seed, and it requires more time for seed maturation. In spite of this, it is possible to achieve seven generations in 1.5 years. Considering the fact that $\mathrm{DH}$ populations of flowering chinese cabbage have not been established because of technical difficulties and genotype dependent recalcitrance, the fast generation procedures developed in this research are a possible alternative for producing pure-line populations. These methods are fast, cost effective, and may have potential applications in breeding and genetic studies of other Brassicaceae vegetables.

\section{Literature Cited}

Ajisaka, H., Y. Kuginuki, and M. Shiratori. 1999. Mapping of loci affecting the cultural efficiency of microspore culture of Brassica rapa L. syn. campestris L. using DNA polymorphism. Breed. Sci. 49:187-192.

Chase, S.S. 1952. Production of homozygous diploids of maize from monoploids. Agron. J. 44:263-267.

Ferrie, A.M.R. and C. Mollers. 2010. Haploids and doubled haploids in Brassica spp. for genetic and genomic research. Plant Cell Tissue Organ Cult. 104:375-386.

Li, G., H. Zhang, H. Huang, Y. Qiao, and Y. Zheng. 2011. Research progress on flowering chinese cabbage breeding in Guangdong province. China Veg. 20:9-14.

Liu, H., P. Zwer, H. Wang, C. Liu, Z. Lu, Y. Wang, and G. Yan. 2016. A fast generation cycling system for oat and triticale breeding. Plant Breed. 135:574-579

Mobini, S.H. and T.D. Warkentin. 2016. A simple and efficient method of in vivo rapid generation technology in pea (Pisum sativum L.). In Vitro Cell. Dev. Biol. Plant 52:530-536.

Mou, B. and G. Wang. 2012. Asia's indigenous horticultural crops: An introduction. HortScience 47:819-820

Murashige, T. and F. Skoog. 1962. A revised medium for rapid growth and bioassays with tobacco cultures. Physiol. Plant. 15:473-497.

Ochatt, S.J., R.S. Sangwan, P.Y. Marget, Y.A. Ndong, M. Rancillac, and P. Perney. 2002. New approaches towards the shortening of generation cycles for faster breeding of protein legumes. Plant Breed. 121:436-440.

Ribalta, F.M., J.S. Croser, W. Erskine, P.M. Finnegan, M.M. Lulsdorf, and S.J. Ochatt. 2014. Antigibberellin-induced reduction of internode length favors in vitro flowering and seed-set in different pea genotypes. Biol. Plant. 58:39-46.

Sato, T., T. Nishio, and M. Hirai. 1989. Plant regeneration from isolated microspore cultures of chinese cabbage (Brassica campestris spp. pekinensis). Plant Cell Rep. 8:486-488.

Sciarappa, W.J., J. Simon, R. Govindasamy, K. Kelley, F. Mangan, S. Zhang, S. Arumugam, P. Nitzsche, R.V. Vranken, S. Komar, A. Ayeni, G. McAvoy, C. Park, W. Reichert, D. Byrnes, Q. $\mathrm{Wu}$, and B. Shilling. 2016. Asian crops overview: Consumer preference and cultivar growth on the east coast of the United States. HortScience 51:1344-1350.

Shumilina, D.V., N.A. Shmykova, L.L. Bondareva, and T.P. Suprunova. 2015. Effect of genotype and medium culture content on microsporederived embryo formation in chinese cabbage (Brassica rapa $\mathrm{ssp}$. chinensis) cv. Lastochka Biol. Bull. 42:302-309.

Stephens, J.M. 2015. Cabbage, chinese Brassica campestris L. (Pekinensis group), Brassica campestris L. (Chinensis group). Horticultural Sciences. IFAS Extension, HS569. <https:// edis.ifas.ufl.edu/mv036>

Wang, T., X. Cai, J. Zhang, H. Li, and Z. Ye. 2010. The culture and early male sterile identification of distant hybrid embryos derived from Brassica oleracea var. capitata L. and male sterile line in B. juncea. Yuan Yi Xue Bao 37:1661-1666.

Yang, S., X. Liu, Y. Fu, X. Zhang, Y. Li, Z. Liu, and H. Feng. 2013. The effect of culture shaking on microspore embryogenesis and embryonic development in Pakchoi (Brassica rapa L. ssp. chinensis). Sci. Hort. 152:70-73.

Zhang, H., Z. Liu, H. Huang, Y. Zheng, and Y. Liu. 2002. Cross-pollination rate in flowering chinese cabbage inbred lines and heterosis combination characteristics. Fifth Youth Academic Assembly of Chinese Society for Horticultural Science, China, 27-29 July 2002.

Zheng, Z., H. Wang, G. Chen, G. Yan, and C. Liu. 2013. A procedure allowing up to eight generations of wheat and nine generations of barley per annum. Euphytica 191:311-316.

Zhu, Y., Q. Liu, C. Wu, and M. Liu. 2008. Study on embryoid induction of Brassica parachinensis Bailey anther culture. Biotechnol. Bul. 2:136-139. 